\title{
Limb salvage arterial surgery for gangrene
}

\author{
G. W. TAYLOR \\ M.S., F.R.C.S. \\ Professor of Surgery and Director of Surgical Professorial Unit, \\ St Bartholomew's Hospital, London
}

IN THIS paper we are concerned with the management of the severely ischaemic state resulting from chronic occlusive arterial disease. For all practical purposes this means atherosclerotic obliteration of the arterial supply to the lower limb. In general, atherosclerosis of the peripheral arteries is a relatively benign disease, and the collateral circulation compensates well for the loss of the main vessel. In about $15 \%$ of patients the disease is more extensive and the degree of ischaemia such that major amputation becomes inevitable unless blood flow to the distal tissues can be improved. Some patients will reach this stage after a period with intermittent claudication alone and others will present de novo with abrupt severe ischaemia due to sudden and extensive thrombosis in their atherosclerotic but previously unobstructed arteries. The latter group may resemble closely the clinical picture of embolic occlusion, but the absence of a central source for embolus, the age of the patient and clinical evidence of atherosclerotic disease in other areas, will usually point to the correct diagnosis.

\section{The symptoms and signs of severe ischaemia}

These are well known, with pain in the forefoot and toes as the dominant feature. This pain is constant and unrelenting and is present with the patient at rest, in contradistinction to the purely exercise discomfort of intermittent claudication. There is no quantitative relationship between the severity of pain and the presence or absence of established tissue necrosis. Ischaemic neuritis has been postulated as the source of the severe pain but this seems unlikely in view of the immediate cessation of this symptom following successful restoration of blood flow.

The severity of the ischaemia may vary from a mild state in which such symptoms as coldness, paraesthesiae and slight discomfort predominate, to impending or established major gangrene when intolerable and constant pain prevents walking, eating or sleeping. In the more severe forms slight relief may be gained from dependency of the affected limb and the patient may hang the leg out of bed at night-time or attempt to sleep sitting semi-upright in a chair. In these circumstances postural oedema quickly develops and the cold, cyanosed, swollen foot has an ominous prognosis. The physical signs vary with the degree of ischaemia. In most limbs there will be clinical evidence of major arterial obstruction with absent distal pulses. In twothirds of patients the femoral pulse alone will be detectable in the affected limb while in the remaining third the disease extends to the aorto-iliac segment and the limb will be pulseless on clinical examination. Auscultation over the open arteries proximal to the occluded segment often reveals a systolic murmur indicative of more proximal disease in the as yet unobstructed area. Palpation will reveal a lower skin temperature of the affected foot and the characteristic changes of colour with postureelevation pallor and dependent rubor-will be present. During elevation the vis a tergo is insufficient to fill the superficial veins and these collapse to form furrows and gutters on the skin surface. In addition to the foregoing, small areas of necrosis may develop on the tips or distal phalanges of one or more toes and, in the more severe states, gangrenous change may extend to involve the forefoot itself. The ischaemic foot tolerates minor trauma poorly and overt gangrenous change may be precipitated by minor chiropody, or slight trauma at pressure sites such as the heel, metatarsal borders of the foot or malleoli.

Above all else, however, pain is the most important symptom and is the motivating reason for therapeutic efforts to improve blood flow. By no means every ischaemic limb will have developed areas of established tissue death and yet ischaemic pain may demand major amputation unless the blood supply can be improved. Conversely in slowly progressive ischaemia areas of digital necrosis may develop gradually and painless dry gangrene of digits in an elderly patient may need little in the way of active treatment.

\section{The arterial lesion}

Atherosclerotic disease of the peripheral arteries is a slowly progressive and sometimes episodic disease. Usually chronic stenosis precedes total occlusion and the pressure gradient engendered by 
the former ensures a developed collateral circulation before the latter event occurs. The resulting outcome is, therefore, only a moderate deficiency in blood flow with intermittent claudication as the chief symptom. With a rapidly developing thrombosis or extensive disease involving multiple segments of the arterial tree the collateral circulation is correspondingly poorer and a more serious degree of ischaemia results. The patient with pregangrene and rest pain is in the latter category and will inevitably show long segmental occlusions of relatively rapid development or multiple areas of disease with a poor collateral network. For example aorto-iliac disease alone rarely causes severe ischaemia but may do so in combination with superficial femoral artery and profunda femoris disease. Each area of disease in a vascular tree constitutes an abnormal resistance to flow and the total flow through such a vascular tree is governed by the segment with maximal resistance ' $R$ max' (Weale, 1966). Arterial surgery can improve flow either by a total by-pass of the diseased segments or by by-pass or reconstruction of the segment responsible for the maximal resistance. The vascular surgeon must be sure, therefore, that he is dealing with ' $R$ max' and careful pre-operative evaluation by complete arteriographic visualization of the vascular tree of the limb is essential.

\section{Treatment}

The aim of treatment is to increase total blood flow through the ischaemic foot. It is worth stressing here that the harmful effects of ischaemia in this context are due to diminution of blood flow through the part and unless this can be corrected it is of little avail to increase one component of the metabolic requirements of tissue. Ischaemia cannot be totally equated with anoxia and hyperbaric oxygen treatment cannot be expected to produce lasting benefit in chronic arterial disease. Systemically administered vasodilator drugs are not only useless in the severely ischaemic state but may cause positive harm by deviation of the circulating blood volume into the open circuits of the vascular tree at the expense of flow in the ischaemic limb (Gillespie, 1959). Treatment, therefore, lies between an attempt at limb salvage by an operation designed to increase blood flow in the ischaemic tissues or major amputation. Theoretically blood flow may be improved surgically either indirectly by lumbar sympathectomy or directly by reconstructive arterial surgery.

\section{The rationale of lumbar sympathectomy}

Sympathectomy releases the physiological component of the peripheral resistance by abolishing vasoconstrictor tone in the cutaneous arterioles. Unfortunately most patients with major arterial occlusion and severe ischaemia will fail to benefit by sympathectomy alone. This is because the patholo-气 gical resistance offered by the main artery block and $\frac{\pi}{\Phi}$ the collateral network is considerably greater than $\stackrel{\varnothing}{\complement}$ the physiological resistance abolished by sympathectomy. In these circumstances ' $R$ max' lies $\overrightarrow{\bar{s}}$ with the immutable resistance offered by the arterialo block and its meagre collaterals and reduction of the $\frac{\bar{O}}{-}$ downstream physiological resistance produces now significant benefit. Sympathectomy alone, therefore, $\mathbb{\Phi}$ is only effective in the less severe forms of major ischaemia and the proper selection of patients for this ${ }^{\infty}$ operation is a problem of delicate clinical appraisalo (vide infra). If, however, ' $R$ max' is abolished by $\overrightarrow{\vec{H}}$ reconstructive surgery then the physiological com- $-\infty$ ponent becomes the controlling factor governingo blood flow and sympathectomy in conjunction with 3 . arterial surgery is a rational and worthwhile exercise. (Terry, Allan \& Taylor, 1970).

\section{Management of necrotic areas}

It is of paramount importance to deal with these lesions at the time of reconstructive arterial surgeryo so as to ensure complete healing in as short a timeas possible. In patients with severe arterial diseasez and poor outflow tracts the arterial reconstruction, although initially successful, may fail within a very ${ }_{0}$ few weeks. If, however, during the period of artergi 1 patency complete epithelialization of amputated excised areas has been obtained then re-occlusiorgiso not necessarily followed by a return of rest pain and gangrene. The end result will be a viable and comfortable limb despite relatively early failure of the arterial surgery. Initial procrastination in the management of necrotic areas may thus jeopardize thes chance of a favourable outcome. The evaluation of arterial surgery for gangrene differs from that obtain ing when intermittent claudication is the indication for operation. In the claudicant, if re-occlusion? occurs, all benefit is lost and symptoms return whereas in gangrene a good result may still obtain 3 In summary of the foregoing we advocate a tripartites. policy in the surgical management of the severely? ischaemic limb, i.e. arterial reconstruction, lumbar sympathectomy and excision of areas of irreversible necrosis. These procedures should be carried out at the same operative session.

\section{Pre-operative investigation}

The most important investigation is arteriography which should ideally delineate the vascular tree wel $\omega$ proximal and distal to the main area of obstruction? In most patients this will mean aortography eithee by the translumbar or femoral approach. Distall the tibial circulation to the level of the malleoli should be visualized, and this may need carefus timing in view of the slow progression of the dye through the scanty collaterals. Most patients wilf 
have generalized atherosclerotic disease and involvement of other areas such as the coronary and carotid arteries should be noted in the general assessment. In patients with multiple areas of disease in the vascular supply to the ischaemic limb identification of ' $R$ max' may be helped by measurement of arterial pressure at different levels in the limb by the ultrasonic probe technique (Allan \& Terry, 1969; Yao, 1970).

\section{Selection of patients for surgical treatment}

In a patient with established rest pain and pregangrene decision-making as to surgical treatment is a staged process. It is assumed that supportive medical treatment, e.g. correction of anaemia, treatment of cardiac decompensation, has been carried out if required. Diabetic patients with atherosclerotic disease and main artery occlusion are managed in a similar way to the non-diabetic. Unfortunately the arterial lesion is usually more severe in the diabetic particularly in the smaller tibial arteries and the prognosis for limb viability is, therefore, correspondingly poorer.

\section{First decision}

(1) Should immediate major amputation be advised?

(2) Should an attempt be made to improve blood flow to the ischaemic limb by surgical means?

In some patients it is at once obvious that amputation is the best solution for such reasons as: (a) extensive irreversible gangrenous change involving the entire foot, (b) limb useless for reasons other than ischaemia, e.g. senile bed-ridden patient, severe arthritis, paralysis following previous stroke.

In these situations decision for amputation can be made on clinical grounds alone and further investigations are unnecessary. It will be noted that chronological age of the patient is not used as a criterion in this decision but rather his functional capacity as regards future locomotor ability. Evidence of myocardial disease (unless severely limiting the patient's ability for ambulation) is not considered as a bar to limb salvage for the alternative is major amputation which carries an equal or greater operative mortality. If the decision for amputation is not reached on the above grounds the next stage is investigation including arteriography. Further assessment is now possible in view of the radiological evidence of the state of the arterial tree. A proportion of patients will then pass into the amputation category because such extensive obliteration of the arterial system renders reconstruction impossible. In a series of 304 limbs with severe ischaemia we found urgent amputation inevitable in seventy-four, largely because of the arteriographic findings of near total main artery occlusion. This figure suggests that salvage is impracticable from the outset in about $25 \%$ of severely ischaemic limbs. The majority of these patients will accept the decision for major amputation with equanimity and with relief at the prospect of freedom from continuing pain. Some, however, cannot immediately reconcile themselves to loss of the limb and in these circumstances it is wise not to press unduly but to wait until spontaneous and ready agreement on the part of the patient is obtained. A more rapid and complete rehabilitation with the prosthetic limb is obtained if the patient approaches the situation with the right motivation from the outset. The first decision then has excluded those patients in whom amputation is inevitable and in the remainder the second decision concerns the type of surgery appropriate to the individual case.

\section{Second decision. Type of surgical treatment}

(a) Sympathectomy alone.

(b) Arterial reconstruction and sympathectomy.

Unfortunately very few limbs can be saved from major amputation by lumbar sympathectomy alone. In the majority of patients with severe ischaemia the pathological resistance of the diseased vascular tree far outweighs the physiological resistance removed by sympathetic ablation. Therefore this operation is only beneficial at the most favourable end of the spectrum encompassing the clinical picture of severe ischaemia. Thus the rest pain should be mild in character and of a degree that is abolished by a few days' bed-rest with a foot-down tilt. If necrosis has already begun it should be confined to the tips or distal phalanges of the toes. Gangrene of whole digits or extension to the dorsum of the foot is unlikely to improve with sympathectomy alone. There is no precise clinical investigation that is of much assistance with this problem and the decision is made largely on clinical grounds. In the correct circumstances, however, sympathectomy may bring permanent relief-and in this connection chemical sympathectomy by paravertebral injection is a valuable technique in skilled hands.

\section{Reconstructive arterial surgery and sympathectomy}

Most patients will require this combination of operative treatment. The precise form of reconstruction will depend on information derived by careful study of adequate arteriography, and generally speaking the major effort will be in either the aortoiliac or the femoro-popliteal areas.

\section{Aorto-iliac reconstruction}

Disease confined to the aorto-iliac segment alone rarely causes severe ischaemia and in this category of patient there are usually additional occlusions in the femoro-popliteal or tibial areas. However, 
revascularization to the common femoral artery is capable of reversing the severe ischaemia in the majority of such patients provided the profunda femoris artery is healthy. Stenosis of the profunda origin is easily missed on the usual $\mathrm{A} / \mathrm{P}$ arteriogram and lateral films of this area should be obtained during the initial aortography (Martin \& Frawley, 1971). If there is significant stenosis at this level it should be corrected at the same sitting as the aortoiliac operation. In our series of 230 reconstructions for severe ischaemia the aorto-iliac segment was the major site of surgery in sixty patients (Table 2). A little over half of these were treated by rebore and the remainder by Dacron by-pass grafts or rarely long vein grafts from the common iliac to the popliteal artery. In patients with aorto-iliac disease and one severely ischaemic limb it is unwise to confine the reconstruction to the common iliac artery on the ischaemic side. So doing may jeopardize the vascular supply to the other limb by pre-operative or postoperative thrombosis in the opposite iliac system. Rebore or by-pass grafting, therefore, normally includes the terminal aorta and both iliac systems. The rebore procedure is best confined to those patients whose disease largely ceases at the common iliac bifurcation and who have good external iliac arteries. This decision cannot always be made accurately on arteriographic assessment alone and is best left until the external iliac arteries are exposed and carefully palpated throughout their length. If these vessels are not in a reasonable condition a Dacron bifurcation graft is led to the common femoral arteries. In a small proportion of patients the condition of the limb will not improve following successful reconstruction to common femoral levels. In this group extension of the revascularization by autogenous vein grafting to a more distal level will be necessary. We prefer to do this as a second stage procedure.

\section{Femoro-popliteal reconstruction}

The majority of patients with severe ischaemia require reconstruction in this area and this was necessary in 170 of the 230 limbs treated in our series. Our preference is for autogenous vein grafts although rebore is applicable to disease mainly confined to the superficial femoral artery. In this context it is rarely practicable to do a short segmental rebore and the entire segment from common femoral to upper popliteal should be dealt with. Patch grafting with Dacron or segments from the long saphenous vein at ankle level (never sacrifice the upper reaches of the long saphenous vein for patch purposes) is usually done at arteriotomy sites and great care is necessary to obtain a good intimal junction at the distal end of the rebore procedure. If the disease extends well into the popliteal segment autogenous vein by-pass is preferable. For the femoro-poplitea segment we use reversed vein grafts inserted between the common femoral artery (which often requires local rebore) to the distal part of the popliteal just above its bifurcation. It is better not to attemp rebore at the distal anastomosis but to use the vein junction as a patch angioplasty to widen the thickeneof popliteal artery. If the long saphenous is unsuitable the cephalic vein is used and is usually long enough to bridge from common femoral to distal poplitea fu levels (Kakkar, 1969). Occasionally a combination ${ }^{s}$ of proximal rebore and distal vein grafting enableș the proximal segment of an otherwise too narrow long saphenous vein to be used.

\section{The run-off vessels}

The effect of the run-off on the outcome of arteria surgery in severe ischaemia has been discussed in a previous report (Taylor, 1964). This study showed® that while a high run-off resistance significantlys increased the incidence of early and late failure of the reconstruction, acceptable limb salvage rates were still obtainable. Patients with severe ischaemia almost never have a totally normal tibial outflows tract. A good result is usual, however, if one tibia artery is open and in free communication with the popliteal artery.

In about half the patients with pregangrene proximal reaches of one or more tibial arteries be patent but occlusion will have taken place at as more distal level. In this category reconstruction to popliteal level, although unsuccessful in restoring कै pedal pulse is often followed by a satisfactory in 2 crease in blood flow through the ischaemic foot $\overline{\overline{0}}$ This is achieved by the increase in perfusion pressure to the distal collaterals following removal of the proximal ' $R$ max' by the reconstructive procedure.

\section{The blind popliteal segment}

In some patients arteriography will reveal the usual femoro-popliteal occlusion and an oper popliteal segment which ends blindly without direce communication with the tibial arteries. This situation does not necessarily preclude implantation of the distal end of a vein graft into the blind segment $\frac{D}{0}$ but the presence of multiple collaterals arising from the popliteal artery and proceeding distally must bes demonstrated on the arteriogram. The situation occasionally arises (particularly so in diabetic patients) that there is such severe tibial arteriar disease that ' $R$ max' is situated in the below-kneo segment and by-pass grafting to knee level, althoughe successful in restoring a popliteal pulse, will not there improve the ischaemic state of the foot. This may result in an inevitable, but galling decision for majors amputation despite a patent by-pass graft. For this reason, with extensive tibial artery disease we prefer $\frac{O}{D}$ 
whenever possible, to extend the by-pass to an open segment of tibial artery in the lower third of the leg.

\section{Femoro-tibial by-pass}

This technique is required for total popliteal or severe proximal tibial arterial occlusion. Our experience has been satisfactory using a patent distal posterior tibial arterial segment, less so with the anterior tibial artery and disappointing when the peroneal is the remaining below-knee artery. For the distal posterior tibial artery we prefer in-situ use of the long saphenous vein, destroying the valves with a vein stripper guide passed retrogradely, and ligation of the major venous tributaries in continuity. It is convenient to turn the distal end of the long saphenous vein into the posterior tibial artery a few centimeters above the medial malleolus.

\section{Cross-over techniques}

In some circumstances it may be advantageous to deviate blood to the ischaemic limb from other open circuits. The cross-over femoro-femoral graft is a good example of this technique, and can be used in predominantly unilateral iliac artery obstruction with a good outflow tract from the distal common femoral artery. An autogenous vein graft or prosthesis is led subcutaneously, across the pubis, from the femoral artery of the normal leg to the femoral artery of the ischaemic limb. This is a comparatively minor operative procedure and is particularly indicated in poor risk patients in whom the alternative aorto-femoral reconstruction would be too great a burden. The axillo-femoral graft is a similar type of procedure in which the axillary and femoral arteries are connected by a graft placed subcutaneously between the axilla and groin. This requires a much longer by-pass than the femoro-femoral application and the long-term patency rate is correspondingly poorer.

\section{Lumbar sympathectomy}

This is usually done at the conclusion of the arterial reconstruction, when the effect of the systemic heparinization is wearing off and while the leg incisions are being closed. In aorto-iliac reconstructions a transperitoneal approach is obviously done and both sides are resected. In femoro-popliteal reconstructions a unilateral sympathectomy is performed by an extraperitoneal muscle splitting approach. An extensive removal of the sympathetic chain is unnecessary and a resection $2 \mathrm{~cm}$ long centred on L4 is quite sufficient.

\section{Management of established necrotic areas}

If there are gangrenous digits or areas of gangrene encroaching on the forefoot or ischaemic ulceration of the lower leg these should be dealt with at the time of reconstruction and lumbar sympathectomy.
Formal amputations are not used and the exercise is merely one of conservative local excision of nonviable areas with whatever technique is required to ensure rapid epithelial cover. This may require partial excision of the tarsus to enable skin flaps to meet. Areas of necrosis involving the lateral border of the foot usually require immediate skin grafting after the excision, as do ischaemic ulcers of the lower leg or malleolar region. Deep ulcers of the heel are most difficult to deal with but usually benefit from saucerization and may then accept a skin graft. On occasion it is impossible to predict the extent of nonviability before arterial reconstruction. In these circumstances excision is delayed for 5 or 6 days post-reconstruction. This time interval allows sharp and accurate demarcation to take place. A delay may also be necessary if there is significant infection in the necrotic areas. Excision is carried out at the initial procedure but closure or skin grafting is postponed until the infection has been overcome.

\section{Results in terms of limb salvage}

Patients with severe ischaemia usually have an aggressive form of arterial disease, are in an elderly age group and have a limited life expectancy. It can be legitimately asked, therefore, as to whether limb salvage surgery is a worthwhile procedure or whether patients would not be better served by immediate amputation. In order to answer this question information on such factors as operative mortality and longterm success rates is required. Analysis of results in 304 severely ischaemic limbs treated at St Bartholonew's Hospital provides this type of data. In this series there were 285 patients and bilateral disease in nineteen gave a total of 304 ischaemic limbs. The age-range of the patients was between 35 and 86 years and in all of them atherosclerosis was the causative disease, and the degree of ischaemia was such that major amputation was indicated if arterial reconstruction was impracticable. In seventy-four $(24 \%)$ limbs immediate amputation was necessary for reasons described earlier in this paper leaving 230 limbs in which the programme of limb salvage arterial surgery was carried out (Table 1). Table 2 sets out the anatomical site of the reconstruction and the operative mortality in each group. Femorotibial grafts are included in the femoro-popliteal segment. Most of the operative mortality resulted from coronary occlusions in the post-operative period. Reconstruction in the leg shows a low

\section{TABle 1.}

\begin{tabular}{lr}
\hline No. of patients & 285 \\
No. of severely ischaemic limbs & 304 \\
Immediate amputation (limbs) & 74 \\
Reconstructive arterial surgery & 230 \\
\hline
\end{tabular}


TABLE 3.

\begin{tabular}{lccrr}
\hline $\begin{array}{c}\text { Total limbs and } \\
\text { follow-up period }\end{array}$ & Amputations & $\begin{array}{c}\text { Died in follow-up } \\
\text { period }\end{array}$ & $\begin{array}{c}\text { Viable limbs in } \\
\text { surviving patients }\end{array}$ & $\begin{array}{c}\text { Patent reconstruction at } \\
\text { follow-up. Surviving patients }\end{array}$ \\
\hline $\begin{array}{l}\text { Aorto-iliac } \\
\text { 1 year (60) }\end{array}$ & 11 & 4 & $45(83 \%)$ & $42(72 \%)$ \\
3 years (43) & 10 & 4 & $29(74 \%)$ & $24(61 \%)$ \\
5 years (22) & 5 & 6 & $11(68 \%)$ & $8(50 \%)$ \\
Femoro-popliteal & & & & \\
1 year (170) & 32 & 12 & $126(80 \%)$ & $101(63 \%)$ \\
3 years (111) & 26 & 21 & $64(71 \%)$ & $81(45 \%)$ \\
5 years (43) & 10 & 14 & $19(65 \%)$ & $11(38 \%)$ \\
\hline
\end{tabular}

TABLe 2.

\begin{tabular}{|c|c|c|}
\hline Type of reconstructio & & $\begin{array}{c}\text { Operative } \\
\text { deaths }\end{array}$ \\
\hline $\begin{array}{l}\text { Aorto-iliac } \\
\text { Thrombo-endarterectomy } \\
\text { Dacron replacement } \\
\text { Autogenous vein graft } \\
\text { Femoro-popliteal* } \\
\text { Thrombo-endarterectomy } \\
\text { Autogenous vein graft }\end{array}$ & $\left.\begin{array}{r}32 \\
24 \\
4\end{array}\right\} 60$ & $3(1.7 \%)$ \\
\hline
\end{tabular}

* Includes femoro-tibial.

operative mortality $(1.7 \%)$ despite the age-group of the patients but aortic surgery proved more taxing with a mortality rate of $5 \%$.

Table 3 displays the results in terms of limb salvage for aorto-iliac and femoro-popliteal reconstructive surgery at 1, 3 and 5 years. Approximately half of these patients required minor amputation for established necrosis and lumbar sympathectomy was done in most of them. It can be seen that the results were comparable in both groups and that long-term viability of limbs was achieved in about two-thirds of surviving patients. It should also be noted that limb viability was maintained until the time of death in those patients who died during the period of observation. If allowance is made for the $25 \%$ immediate amputation rate in the series it can be stated as an approximation that about half the limbs presenting with severe ischaemia can be salvaged by arterial surgery, and will remain viable and useful over a 5-year period. The final column in
Table 3 expresses the patency rate of the arterial reconstruction in surviving patients. Comparison with the limb viability rate in the neighbouring column shows that a significant proportion of limbs remained viable despite late failure of the arterial surgery. This is particularly noticeable in the femoropopliteal group where, for example, of the nineteen limbs viable at 5 years eight had survived arterial of re-occlusions. The phenomenon of limb survival 0 despite failure of reconstruction applies only to reocclusions occurring after the first few post-operative weeks. Primary failure of the arterial surgery leaves the limb in the pre-operative state and major $\vec{\omega}$ amputation is inevitable.

\section{References}

Allan, J.S. \& TerRy, H.J. (1969) The evaluation of añ ultrasonic flow detector for the assessment of peripheral vascular disease. Cardiovascular Research, 3, 503.

GillesPIE, J.A. (1959) The case against vasodilator drugs in occlusive vascular disease of the legs. Lancet, 2, 995.

KAKKAR, V.V. (1969) The cephalic vein as a peripheral vascular graft. Surgery, Gynaecology and Obstetrics, 128, 551.

Martin, P. \& Frawley, J. (In press.)

TAYLOR, G.W. (1964) Arterial surgery for major gangrene. Journal of Cardiovascular Surgery, 5, 523.

TerRy, H.J., Allan, J.S. \& TAYlor, G.W. (1970) The effect of adding lumbar sympathectomy to reconstructive arterial surgery in the lower limb. British Journal of Surgery, 57, 51 .

Weale, F.E. (1966) An Introduction to Surgical Haemodynamics. p. 49. Lloyd-Luke, London.

YAO, S.T. (1970) Haemodynamic studies in peripheral arterial disease. British Journal of Surgery, 57, 761. 